\title{
Minimally invasive approaches: cryoablation
}

\author{
T. L. Huston, R. M. Simmons \\ Department of Surgery, Weill-Cornell Medical Center, New York-Presbyterian Hospital, \\ New York, NY, USA.
}

\begin{abstract}
As widespread screening for breast cancer detects more women at younger ages and earlier stages, the need for minimally invasive, cosmetically preferable approaches to its treatment grows. The combination of a superficial location on the thorax and lack of intervening organs makes the breast ideal for ablative therapies, one of which is cryoablation. Cryoablation destroys tissue through multiple cycles of localized freezing. Major advantages to cryoablation are: the ability to perform the procedure in the office under real-time ultrasound guidance, improved post-procedure cosmesis when compared to breast conserving surgery since only a $2 \mathrm{~mm}$ skin incision is necessary, and the avoidance of anesthesia since freezing numbs the breast. Numerous studies, reviewed here, have demonstrated success in eradication of invasive breast cancer using this modality. However, before cryoablation can be added to the armamentarium of those who treat breast cancer, prospective randomized trials are needed to determine its long-term effectiveness.
\end{abstract}

Keywords: Ablation; Breast cancer; Cryoablation; Minimally invasive surgery

\section{Introduction}

The American Cancer Society projects over 211000 new cases of invasive breast cancer will be diagnosed in the USA in 2005 [1]. Tumors under $2 \mathrm{~cm}$ demonstrate the steepest increase in incidence, due to improvements in mammographic screening [2]. Overall, one-third of all new cancers are less than $1 \mathrm{~cm}$ [3]. Specifically, these T1a and T1b tumors have an excellent prognosis, with approximately $90 \%$ of node negative patients alive 18 years later [4]. For patients with one to three positive nodes and T1 tumors, the 20 -year disease free survival rate is $81 \%$ [5]. Widespread screening for breast cancer, detecting more women at younger ages and earlier stages coupled with improved survival, is increasing the demand for minimally invasive, cosmetically preferable treatment modalities.

Correspondence to: Rache M. Simmons, 425 East 61st Street, 8th floor, New York, NY 10021, USA. E-mail: rms2002@med.cornell.edu; Tel: +1 212821 0853; Fax: +1212 8210823

Publication date 31/08/05 BCO/373/2005/FO
For decades, ablative techniques have been successfully used to treat metastatic hepatic tumors. More recently, they have been applied to malignancies in the lung, bone, central nervous system, kidney, prostate gland and pancreas [6]. The breast is an ideal model for ablative therapies owing to its superficial location on the thorax and absence of intervening organs between it and the skin. Ablative therapies studied on the breast include cryoablation, radiofrequency ablation, interstitial laser ablation, focused ultrasound ablation and microwave thermotherapy. This overview will focus on cryoablation.

\section{How cryoablation works}

Cryoablation is the only ablative therapy that does not use heat. It destroys tissue through multiple cycles of localized freezing. The target temperature with each cycle for effective tissue destruction is as low as $-160^{\circ} \mathrm{C}$ to $-190^{\circ} \mathrm{C}[7-9]$. The overall volume and dimensions of the tumor determine the number of necessary cycles for effective ablation [7-11]. It has 
been shown that gross determination of tissue death is difficult the first week following the procedure. Therefore, if post-procedural resection is planned, it is recommended to wait at least 1 week in order to obtain adequate resection margins and perform an accurate histological analysis [7].

One of the major advantages to cryoablation is that it can be performed in the outpatient setting with very little discomfort to the patient. A standard prerequisite is a core biopsy of the breast carcinoma to determine histological type, differentiation, presence of estrogen and progesterone receptors, HER-2/neu, and markers of proliferation. Once the tumor has been destroyed, none of these markers can be reliably assessed [7]. In order to cryoablate a tumor, it is located by ultrasound and the overlying skin numbed with a local anesthetic. A small $2 \mathrm{~mm}$ skin incision is made with a scalpel and the cryoprobe is inserted. The cryoprobe itself is entirely insulated except for a small, sharp area at the tip placed directly into the tumor. Since the freezing procedure itself acts as an anesthetic on the breast tissue, no additional anesthesia beyond the intradermal injection is needed. Patients stay completely awake and are able to view the procedure on the ultrasound monitor along with their physician [10]. On ultrasound, the sonographic freezeball created by the liquid nitrogen or argon gas can be clearly seen, due to a highly echogenic interface between frozen and unfrozen tissue.

Early pilot studies demonstrated thermal burns to the skin from freezeballs which were too superficial. Saline is therefore injected into the breast tissue between tumor and skin to create a separation and maintain a suitable distance. Alternatively, room temperature saline or water can be dripped directly onto the skin's surface to protect it [10]. Saline can also be used to create a safe margin between the pectoralis muscle and a deep breast tumor.

\section{Studies supporting cryoablation}

The first documented use of cryoablation in the treatment of breast cancer was published in the late 1980s. The case was that of a 77 -year-old woman with a $2 \mathrm{~cm}$ palpable mass, which appeared malignant on mammogram. Following cryoablation and resection, no viable tumor cells were identified in the pathologic specimen. The patient had a brief and uneventful recovery and, 2 years later, was clinically and mammographically disease free [12].

Cryoablation without resection has been documented once, thus providing the only example of the natural history of cryoablated breast cancers. The patient was a 76-year-old woman with two foci of infiltrating lobular carcinoma $(0.5$ and $0.8 \mathrm{~cm})$ in the same quadrant. Pre-procedurally, the diagnosis was confirmed by ultrasound guided core needle biopsy. Cryoablation was performed separately on both tumors via sonographic guidance and the masses were not resected. Core needle biopsy at 4 and 12 weeks post-ablation revealed tissue necrosis, inflammatory cells and cellular debris, but failed to show persistent tumor. Shortly after the procedure, the patient developed a $2 \mathrm{~cm}$ firmness in the area between the two cryoablation zones, which spontaneously resolved within 3 months [9].

Numerous groups have shown that ductal carcinoma in situ (DCIS) is difficult to fully eradicate using cryoablation. Stocks published a series of 11 women with invasive breast cancer who underwent cryoablation followed by resection within 1-3 weeks [13]. In this study, mean tumor size was $1.3 \mathrm{~cm}$ (range $0.7-2.2 \mathrm{~cm}$ ). In each case, a sonographic freezeball surrounded the tumor as well as a $1 \mathrm{~cm}$ margin of normal appearing breast parenchyma. Ten of 11 (91\%) tumors showed complete ablation. One tumor demonstrated residual DCIS at the margin of the ablation zone [13]. Pfleiderer's series included 15 women with 16 breast cancers, averaging $2.1 \mathrm{~cm}$ [14]. The mean freezeball diameter was $2.8 \mathrm{~cm}$ and all tumors were resected 5 days following cryoablation. No severe side effects were observed. The five tumors less than $1.6 \mathrm{~cm}$ had no evidence of invasive cancer after treatment, however two did have DCIS in the surrounding non-ablated breast parenchyma. In 11 tumors equal to or greater than $2.3 \mathrm{~cm}$, histologic examination revealed incomplete necrosis. Overall, these studies both demonstrate how invasive components of small tumors can be adequately treated using cryotherapy. However significant amounts of in situ carcinoma, which may not be detected prior to ablation, represent a relative contraindication to cryoablation [14].

A study from the University of Michigan performed cryoablation on 29 women with core biopsy proven, ultrasound visible invasive breast cancers measuring $2 \mathrm{~cm}$ or less [14]. The cryoablation procedure was followed by planned surgical resection between 1 week and 1 month later. Pathology revealed that all tumors less than $1 \mathrm{~cm}$ were fully ablated. However, in primary tumors measuring $1-1.5 \mathrm{~cm}$, only those without an extensive intraductal component were destroyed. Tumors over $1.5 \mathrm{~cm}$ were not reliably eradicated with cryoablation. Specifically, it was found that DCIS without calcifications resulted in the most treatment failures in the patients with larger tumors. No patient had pain severe enough to require postprocedural narcotics. This study successfully demonstrated that cryoablation is safe and effective, and recommend it be limited to invasive ductal cancers $1.5 \mathrm{~cm}$ or less containing less than $25 \%$ DCIS on core biopsy [15]. 
Tafra and colleagues recently showed how the cryoprobe can used as an alternative to needle localization in order to obtain more accurate margins when excising small, non-palpable, ultrasound-visible tumors [16]. In 24 procedures, a cryoprobe was used to create a freezeball encompassing the tumor along with $0.5-1 \mathrm{~cm}$ of sonographically normal surrounding parenchyma. On pathologic sectioning, tumor sizes ranged from 0.7 to $2 \mathrm{~cm}$ with an average freezeball margin of $0.8 \mathrm{~cm}$ in all directions. Among patients with at least a $0.6 \mathrm{~cm}$ rim of cryoablated tissue beyond the tumor, the margin re-excision rate was only $5.6 \%$. This group concluded that cryoprobeassisted lumpectomy is a viable alternative to the preoperative wire localization because it facilitates an easier and more precise resection while decreasing the incidence of positive margins.

A group at the University of Michigan published the first series detailing mammographic and ultrasonographic findings of breast cancers at cryoablation in order to compare them with the presence of residual malignancy after treatment [17]. In nine women with tumors averaging $1.2 \mathrm{~cm}$, cryoablation was performed followed by lumpectomy 3 weeks later. No residual cancer was identified in tumors $1.7 \mathrm{~cm}$ or smaller, nor in cancers without spiculated margins on ultrasound. Post-ablation mammograms revealed increased density while post-ablation ultrasounds were remarkable for increased echogenecity at the site of the original cancer. These radiologic findings correlated precisely with the known location of the cancer on the pre-procedure imaging. Thus, these authors were the first to report that mammographic density as well as ultrasonographic hyperechogenecity may be indicators of the likelihood of complete ablation [17]. The use of imaging modalities to confirm complete eradication of the breast cancer, in place of the current postprocedural resection, is a step further towards the minimally invasive treatment of breast cancer.

At the Quebec City University Hosptial, a phase I study was carried out in 25 patients in order to determine the feasibility of cryoablation under magnetic resonance (MR) imaging for the treatment of invasive breast cancer [18]. With the near-real-time MR guidance of a $0.5 \mathrm{~T}$ open-configuration MR system, cryoablation was performed 4 weeks prior to scheduled mastectomy. Ablation was complete in 13 of the 25 treated tumors. Combining peri-procedural MR images with post-procedure mammographic and scintigraphic findings enabled a $96 \%$ rate of correctly predicting the results of cryoablation. These researchers found the major drawback to be that the palpable iceball remained in the breast for the entire 4 weeks complicating physical exam and further breast imaging. There were no complications related to the cryosurgery of the use of MR guidance [18].

\section{Conclusion}

These small studies indicate that cryoablation of breast cancers is a promising technique; however, more investigation is needed. It has been shown that the presence of DCIS at the margin of the ablation zone resulted in incomplete tumor necrosis. Thus, patients with DCIS may not be good candidates for cryoablation. Since cryoablation procedures are generally guided by ultrasound, this may represent the limitation of sonography to define DCIS at the perimeter of the malignancy. Major advantages to this technique are the ability to perform it in the office under real-time ultrasound guidance and the avoidance of anesthesia administration as freezing numbs the breast. In addition, there is improved post-procedure cosmesis when compared to breast conserving surgery since only a $2 \mathrm{~mm}$ skin incision is necessary. This enhanced cosmesis is especially true in cases where imaging can be used to determine the effectiveness and post-ablation resection is not necessary. However, prospective, randomized trials are needed to determine the long-term effectiveness of cryoablation for breast cancer.

\section{References}

1. Jemal A, Murray T, Ward E, et al. Cancer statistics, 2005. CA Cancer J Clin 2005; 55: 10-30.

2. Ghafoor A, Jemal A, Ward E, et al. Trends in breast cancer by race and ethnicity. CA Cancer J Clin 2003; 53 342-355.

3. Korourian S, Klimberg S, Henry-Tillman R, et al. Assessment of proliferating cell nuclear antigen activity using digital image analysis in breast carcinoma following magnetic resonance-guided interstitial laser photocoagulation. Breast J 2003; 9: 409-413.

4. Rosen PP, Groshen S, Saigo PE, et al. Pathological prognostic factors in stage I (T1NOM0) and stage II (T1N1M0) breast carcinoma: a study of 644 patients with median follow-up of 18 years. J Clin Oncol 1989; 7: 1239-1251.

5. Quiet CA, Ferguson DJ, Weichselbaum RR, Hellman S. Natural history of node-positive breast cancer: the curability of small cancers with a limited number of positive nodes. J Clin Oncol 1996; 14: 3105-3111.

6. Mirza AN, Fornage BD, Sneige N, et al. Radiofrequency ablation of solid tumors. Cancer J 2001; 7: 95-102.

7. Simmons RM, Dowlatshahi K, Singletary SE, Staren ED. Ablative therapies for breast cancer. Contemp Surg 2002; 58: 61-72.

8. Rand RW, Rand RP, Eggerding FA, et al. Cryolumpectomy for breast cancer: an experimental study. Cryobiology 1985; 22: 307-318.

9. Staren ED, Sabel MS, Gianakakis LM, et al. Cryosurgery of breast cancer. Arch Surg 1997; 132: 28-33.

10. Kaufman CS, Bachman B, Littrup PJ, et al. Office based ultrasound-guided cryoablation of breast fibroadenomas. Am J Surg 2002; 184: 394-400.

11. Rui J, Tatsutani KN, Dahiya R, Rubinsky B. Effect of thermal variable on human breast cancer in cryosurgery. Breast Cancer Res Treat 1999; 53: 185-192. 
12. Rand RW, Rand RP, Eggerding FA, et al. Cryolumpectomy for carcinoma of the breast. Surg Gynecol Obstet 1987; 165: 392-396.

13. Stocks LH, Chang HR, Kaufman CS, et al. Pilot study of minimally invasive ultrasound-guided cryoablation in breast cancer. Am Soc Breast Surg 2002.

14. Pfleiderer SO, Freesmeyer MG, Marx C, et al. Cryotherapy of breast cancer under ultrasound guidance: initial results and limitations. Eur Radiol 2002; 12: 3009-3014.

15. Sabel MS, Kaufman CS, Whitworth PW, et al. Cryoablation of early stage breast cancer: work in progress report of a multi-institutional trial. Ann Surg Oncol 2004; 11: 542-549.
16. Tafra L, Smith SJ, Woodward JE, et al. Pilot trial of cryoprobe-assisted breast-conserving surgery for small ultrasound-visible cancers. Ann Surg Oncol 2003; 10: 1018-1024.

17. Roubidoux MA, Sabel MS, Bailey JE, et al. Small $(<2.0 \mathrm{~cm})$ breast cancers: mammographic and US findings at USguided cryoablation - initial experience. Radiology 2004; 233: 857-867.

18. Morin J, Traore A, Dionne G, et al. Magnetic resonanceguided percutaneous cryosurgery of breast carcinoma: technique and early clinical results. Can J Surg 2004; 47: 347-351. 